\title{
A Review of Online Rehabilitation Protocols Designated for Rotator Cuff Repairs
}

\author{
Reed G. Coda, B.S., Sana G. Cheema, B.A., Christina A. Hermanns, B.S., \\ Armin Tarakemeh, B.A., Matthew L. Vopat, M.D., Meghan Kramer, D.P.T., \\ John Paul Schroeppel, M.D., Scott Mullen, M.D., and Bryan G. Vopat, M.D.
}

\begin{abstract}
Purpose: To compare publicly available rehabilitation protocols designated for rotator cuff (RTC) repairs published online by academic residency programs and private practice institutions. Methods: A systematic electronic search using the Fellowship and Residency Electronic Interactive Database Access System (FREIDA) was performed for RTC repair rehabilitation protocols. Private practice programs with published rehabilitation protocols that were discovered during the Google search were also included for review, but no comprehensive search for private practice protocols was performed. The main exclusion criteria consisted of non-English-language protocols and protocols without any of the time-based components in question. Included protocols were assessed independently based on the specified RTC tear size (small $[\leq 1 \mathrm{~cm}]$, medium [1-4 cm], large or massive [ $\geq 5 \mathrm{~cm}]$, or no mention of size). Protocols were compared based on the inclusion, exclusion, and timing of certain rehabilitation components. Results: A total of 96 rehabilitation protocols were included for review, from 39 academic institutions and 28 private practice programs. Specific instructions for concomitant biceps tenodesis were included in 26 protocols $(27.1 \%)$. Of the 96 protocols, $88(91.7 \%)$ did not place restrictions on early postoperative passive range of motion (PROM) of the shoulder. Isolated PROM with restrictions on active range of motion was most commonly recommended for the first 4 or 6 weeks postoperatively $(80.2 \%)$. Use of a sling or immobilizer was most frequently recommended for the first 4 or 6 weeks postoperatively $(78.1 \%)$. Wide variation was noted in recommendations for returning to resistance strengthening, with the highest incidence being 27 protocols recommending returning at 12 weeks $(28.1 \%)$; this further varied based on the size of the tear. A total of 21 protocols $(21.9 \%)$ recommended the use of cryotherapy postoperatively. Conclusions: Although certain rehabilitation components were common, such as duration of PROM and sling or immobilizer use, a large degree of variation remains among published rehabilitation protocols after RTC repair, and this variability is still seen even when subdividing by the size or severity of the RTC tear. Clinical Relevance: Rehabilitation after RTC repair is crucial to patient outcomes. This study summarizes the variability among online rehabilitation protocols for RTC repair in the United States and emphasizes the importance of appropriate rehabilitation after RTC surgery.
\end{abstract}

$\mathbf{R}$ otator cuff (RTC) tears are a common orthopaedic diagnosis, appearing in patients ranging from young athletes to elderly individuals. Although RTC

From the University of Kansas School of Medicine, Kansas City, Kansas, U.S.A. (R.G.C., S.G.C., C.A.H., A.T., M.K., J.P.S., S.M., B.G.V.); and University of Kansas School of Medicine-Wichita, Wichita, Kansas, U.S.A. (M.L.V.).

The authors report no conflicts of interest in the authorship and publication of this article. Full ICMJE author disclosure forms are available for this article online, as supplementary material.

Received October 6, 2019; accepted March 26, 2020.

Address correspondence to Bryan G. Vopat, M.D., 3901 Rainbow Blvd, Kansas City, KS 66160,U.S.A. E-mail:bvopat@kumc.edu

(C) 2020 THE AUTHORS. Published by Elsevier Inc. on behalf of the Arthroscopy Association of North America. This is an open access article under the CC BY-NC-ND license (http://creativecommons.org/licenses/by-nc-nd/4.0/). 2666-061X/191202

https://doi.org/10.1016/j.asmr.2020.03.006 injuries are common in young athletes with acute injuries or repetitive overhead motions, the incidence of RTC tears tremendously increases with age-related degenerative changes. ${ }^{1-4}$ Treatment of RTC tears is typically individualized based on a variety of factors, such as age; size, severity, and location of the tear; comorbidities; activity level; degree of impairment; and patient expectations. ${ }^{1}$ Both nonoperative and operative treatments are effectively used for pain relief and restoration of shoulder range of motion (ROM) after an RTC tear. 5

Regardless of whether nonoperative or operative intervention is used, rehabilitation of the RTC tendons or muscles and surrounding shoulder girdle plays an essential role in improvement of shoulder function, pain, and overall quality of life. ${ }^{1,7}$ The primary goals of postoperative rehabilitation are protection of the 
healing process and prevention of joint stiffness and muscle atrophy. ${ }^{8}$ Passive range of motion (PROM) in the early postoperative stages is typically performed to the patient's tolerance, not pushing to increase ROM at end ranges but using gentle oscillations to decrease muscular tightness, guarding, and pain.

Because of the heterogeneity in patient demographic characteristics and tear characteristics, both physicians and physical therapists have difficulty in developing postoperative rehabilitation guidelines after RTC repair, thus resulting in a variety of recommendations. ${ }^{9}$ Furthermore, a study performed by Mollison et al. ${ }^{9}$ looking at postoperative rehabilitation after RTC repair called for future studies to evaluate the printed rehabilitation guidelines that surgeons distribute to their patients and therapists. Therefore, the purpose of this study was to compare publicly available rehabilitation protocols designated for RTC repair published online by academic residency programs and private practice institutions. We hypothesized that there would be high variability in postoperative rehabilitation protocols depending on the patient's age, patient's activity level, and/or severity or size of the tear.

\section{Methods}

Using the Fellowship and Residency Electronic Interactive Database Access System (FREIDA), we obtained a comprehensive list of publicly available academic residency programs. By use of this list, an electronic search via Google's search engine (www. google.com) was performed using the following search phrase: "[Program/affiliate hospital/affiliate medical school name] rotator cuff repair rehabilitation protocol." The search was performed on May 2, 2019. In addition to academic institutions, private practice organizations with published RTC repair rehabilitation protocols that appeared during the electronic search were examined to determine whether they fit the inclusion criteria. References were limited to the first page of search results. Figure 1 shows the flow diagram used for this study.

Protocols were included if they were written in the English language and specified rehabilitation after RTC repair. Included protocols were further separated into groups if the protocols indicated rehabilitation for a specific size of RTC tear (small $[\leq 1 \mathrm{~cm}]$, medium [1-4 cm], large or massive [ $\geq 5 \mathrm{~cm}]$, or no mention of size). Protocols were excluded if they were not written in the English language, did not specify rehabilitation after an RTC repair, and/or did not include any of the time-based components in question (ROM, time spent in a sling or immobilizer, time until strengthening, time until initiation of physical therapy, and return to throwing and/or sport). A comprehensive list of the included protocols is presented in Table 1.
The following rehabilitation components were used to compare and contrast the rehabilitation protocols: ROM, time spent in a sling or immobilizer, time until initiation of strengthening of the RTC, return to throwing, return to sport (RTS), use of cryotherapy, inclusion of concomitant biceps tenodesis instructions, and time until initiation of physical therapy. The primary outcome of this study was the timing of each rehabilitation component, as well as the incidence, inclusion, or exclusion of the aforementioned components.

When comparing and contrasting ROM and time spent in a sling or immobilizer, we used the initial number if the protocol specified a range of time. For example, if the protocol specified PROM for 4 to 6 weeks, 4 weeks was included as the data point. For time spent in a sling or immobilizer, the latter number was used if the protocol specified a range. For example, if the protocol specified 4 to 6 weeks of brace wear, 6 weeks was included as the data point. Although many protocols recommended immediate active range of motion (AROM) of the cervical spine, elbow, fingers, and hands, this study only assessed recommendations for shoulder ROM. Likewise, many protocols recommended strengthening of the scapula, biceps, and triceps, but this study focused only on resistance strengthening of the RTC or shoulder.

\section{Results}

Of the 189 academic institutions included in the FREIDA (Fellowship and Residency Electronic Interactive Database Access System) online residency program database, a total of 39 academic institutions and 28 private practice programs had publicly available rehabilitation protocols designated for RTC tears. Of the 39 academic institutions, $12(30.8 \%)$ had multiple protocols depending on the size of the RTC tear. Of the 28 private practice programs, $10(35.7 \%)$ had multiple protocols depending on the RTC tear size. In total, 96 rehabilitation protocols were deemed to fit the inclusion criteria. Of these 96 protocols, only $26(27.1 \%)$ included specific instructions for concomitant biceps tenodesis. These instructions indicated only PROM of the affected elbow for a certain period, ranging from 3 to 10 weeks based on the protocol. In addition, only 21 of 96 protocols $(21.9 \%)$ recommended use of cryotherapy postoperatively.

\section{Range of Motion}

PROM, AROM, and the goal for returning to full ROM of the shoulder were compared at different time intervals postoperatively. Of the 22 protocols that specified large or massive RTC tears, $2(9.1 \%)$ recommended no shoulder ROM within the first 4 weeks and $1(4.5 \%)$ recommended no shoulder ROM within the first 6 weeks. The remaining 19 protocols $(86.4 \%)$ had 
Fig 1. Flow diagram of study. (FREIDA, Fellowship and Residency Electronic Interactive Database Access System; RTC, rotator cuff tear.)

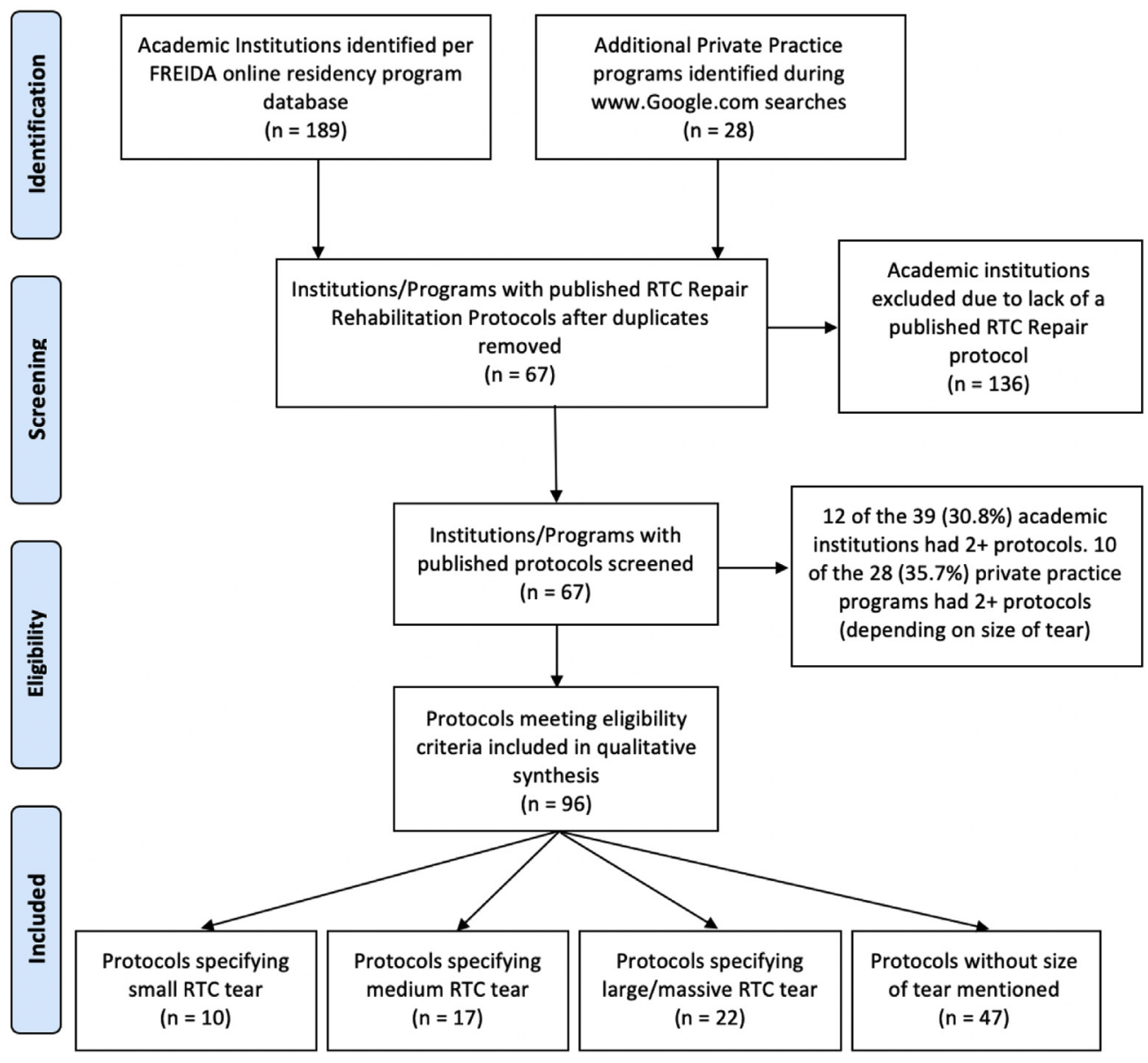

no restrictions on early mobilization. Regarding the recommended duration of PROM from the date of surgery (Fig 2A), protocols ranged from 4 to 10 weeks, with the predominant recommendation, by 10 protocols $(45.5 \%)$, being 6 weeks of PROM.

Of the 17 protocols specific to medium-sized RTC tears, $0(0 \%)$ had restrictions on early postoperative mobilization of the shoulder. For the duration of initial PROM (Fig 2B), 8 protocols $(47.1 \%$ ) recommended PROM for the first 4 weeks postoperatively whereas $6(35.3 \%)$ recommended PROM for the first 6 weeks. Of the 10 protocols specific to small RTC tears, $9(90 \%)$ had no restrictions on early mobilization whereas 1 recommended no shoulder ROM for the first 2 weeks. Of these 9 protocols, $5(50 \%)$ recommended PROM for the first 4 postoperative weeks and $3(30 \%)$ recommended PROM for the first 3 weeks (Fig 2C).

Finally, PROM was compared in the 47 protocols without mention of a specific RTC tear size. Of these 47 protocols, $43(91.5 \%)$ recommended immediate postoperative PROM whereas $2(4.3 \%)$ recommended no ROM for the first 2 postoperative weeks and $2(4.3 \%)$ recommended no ROM for the first 4 postoperative weeks. Regarding the duration of PROM (Fig 2D),
22 protocols $(46.8 \%)$ recommended PROM for the first 4 postoperative weeks and 19 protocols $(40.4 \%)$ recommended PROM for the first 6 postoperative weeks.

The recommended time to transition from PROM to AROM postoperatively was compared throughout the various rehabilitation protocols (Fig 3). Of the 22 protocols that specified large or massive RTC tears (Fig 3A), $10(45.5 \%)$ recommended transitioning to AROM at 8 weeks whereas $6(27.3 \%)$ recommended transitioning at 6 weeks. Regarding the protocols specifying medium RTC tears (Fig 3B), most ( 10 protocols, $58.8 \%$ ) recommended transitioning at 6 weeks postoperatively. When we looked at the protocols specific to small RTC tears (Fig 3C), 4 protocols $(40 \%)$ recommended transitioning at 4 weeks postoperatively and 4 other protocols $(40 \%)$ recommended transitioning at 6 weeks postoperatively. Finally, of the protocols without a specified RTC size (Fig 3D), 30 (63.8\%) recommended that the transition begin at 6 weeks postoperatively. Overall, the time until transition to AROM was directly correlated with the size of the RTC tear (the smaller the tear, the earlier the transition).

Last, the goal for returning to full ROM was compared among the various rehabilitation protocols (Fig 4). 
Table 1. Protocols Included in Study

Large or massive tear protocols

Baylor College of Medicine

Colorado University

Crystal Clinic Orthopaedic Center: physician 1

Crystal Clinic Orthopaedic Center: physician 2

Fowler Kennedy Sports Medicine

Gunderson Health System

Highland Clinic

Jackson Orthopedic Specialists

Lahey Hospital and Medical Center

Lake Cook Orthopedics

Miami Institute for Joint Reconstruction

Northwestern Medical Center

Ohio State University

Ortho Illinois

Ortho NY

Rutgers North Jersey Orthopaedic Institute

Sanford Orthopedics and Sports Medicine

UConn Musculoskeletal Institute

University of Florida

University of Texas Health-Dr. Fullick

University of Wisconsin

VCU Sports Medicine Clinic

Medium tear protocols

Crystal Clinic Orthopaedic Center

Gunderson Health System

Jackson Orthopedic Specialists

Lahey Hospital and Medical Center

Lake Cook Orthopedics

Miami Institute for Joint Reconstruction

Northwestern Medical Center

Ohio State University

Ortho Illinois

Ortho NY

Rutgers North Jersey Orthopaedic Institute

Sanford Orthopedics and Sports Medicine

Tallgrass Orthopedics and Sports Medicine

University of Florida

VCU Sports Medicine Clinic

University of Wisconsin

Small tear protocols

Colorado University

Fowler Kennedy Sports Medicine

Harvard Medical School-Beth Israel Deaconess Medical Center

Highland Clinic

Jackson Orthopedic Specialists

Lake Cook Orthopedics

Northwestern Medical Center

UConn Musculoskeletal Institute

University of Florida

University of Texas Health-Dr. Fullick

Protocols without mention of tear size

Alpine Orthopaedics

Baylor College of Medicine

Boston Sports Medicine

Midwest Orthopaedics at Rush-Dr. Brian Cole

Brigham and Women's Hospital

Brown University-University Orthopedics

Center Orthopedics and Sports Medicine

Chicago Orthopedics and Sports Medicine

Concord Orthopaedics

Connecticut Children's Medical Center

Crystal Clinic Orthopaedic Center
Table 1. Continued

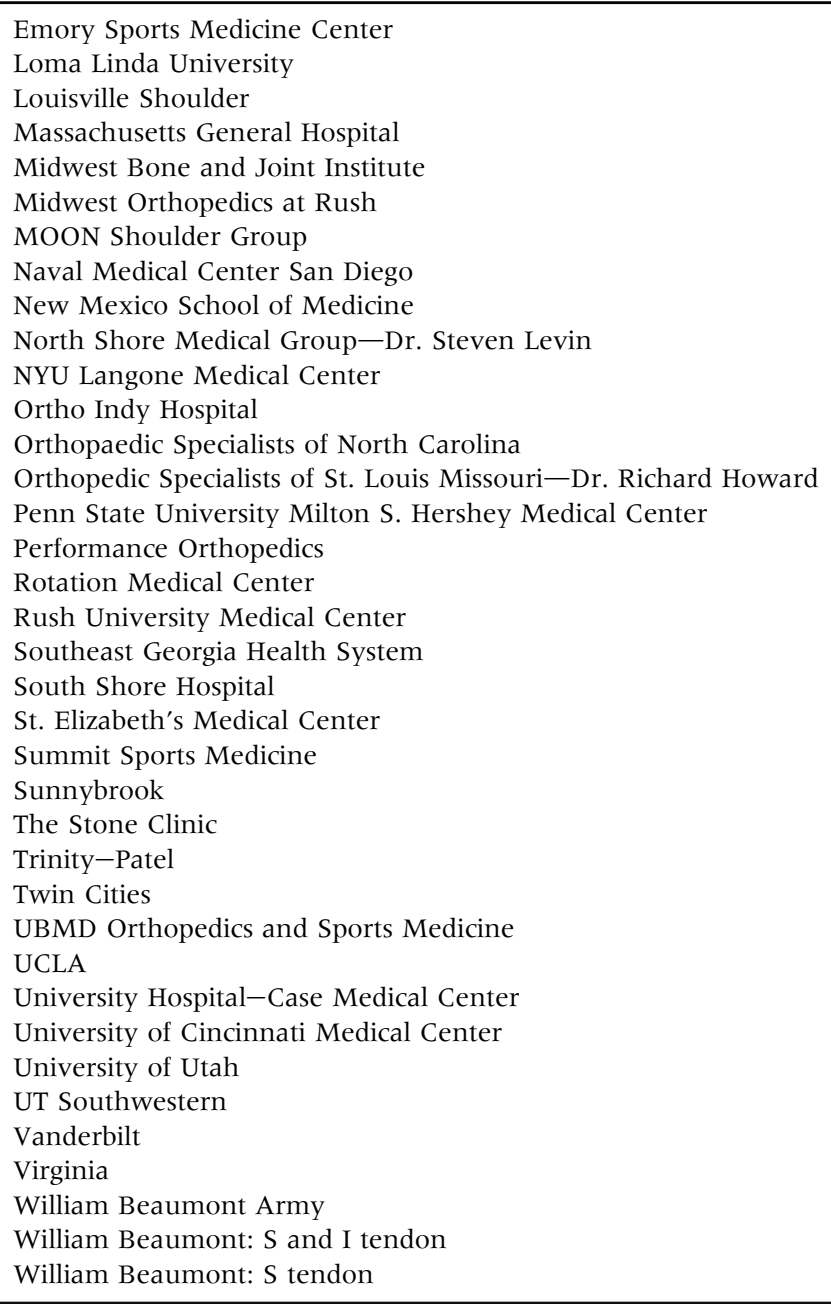

Regarding large or massive RTC protocols (Fig 4A), most (14 protocols, $63.6 \%$ ) recommended returning to full ROM at 3 months. When we compared medium-sized RTC tear protocols (Fig 4B), 9 protocols (52.9\%) recommended returning to full ROM by 12 weeks whereas $6(35.3 \%)$ recommended a full return by 10 weeks. Next, 9 of the 10 protocols $(90 \%)$ specific to small RTC tears made recommendations for returning to full ROM (Fig 4C). The recommendations ranged from 6 to 14 weeks, with the highest incidence being 3 protocols $(30 \%)$ recommending returning by 12 weeks ( 3 months). Finally, when we compared protocols without a specified RTC tear size (Fig 4D), 44 of 47 (93.6\%) made specific recommendations for returning to full ROM. The recommendations ranged from 6 to 16 weeks (4 months), but most protocols (26 protocols, $55.3 \%$ ) recommended returning to full ROM by 12 weeks ( 3 months).

\section{Time Spent in Sling or Immobilizer}

The recommended duration of time spent in a sling or immobilizer in each protocol was evaluated (Fig 5). 


\section{Passive Range of Motion Recommendations}

Fig 2. Passive range - of motion recommendations in protocols designated for large or massive (A), medium (B), and small $(C)$ rotator cuff tears and protocols with rotator cuff size unspecified (D).

\section{A}

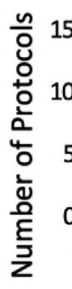

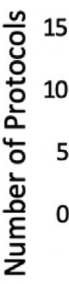

C

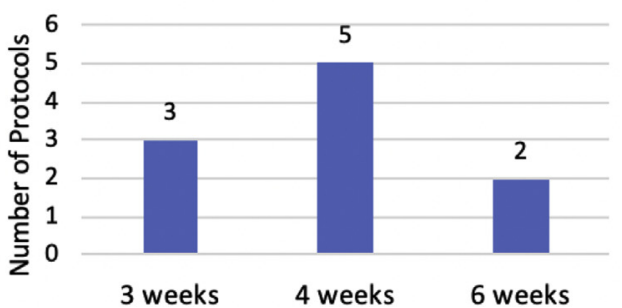

B

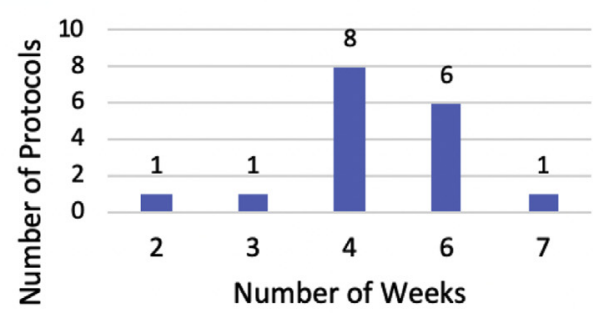

D

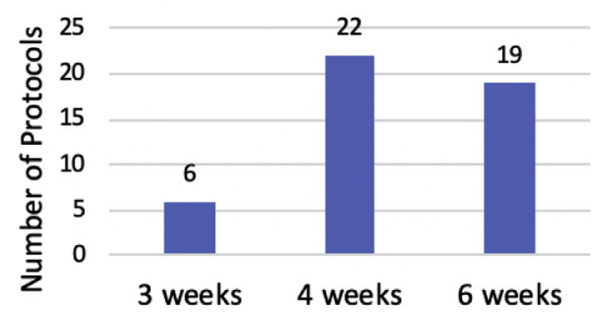

Regarding large to massive tears (Fig 5A), the recommended time spent ranged from 4 to 10 weeks, with most protocols ( 12 protocols, $54.5 \%$ ) recommending use of a sling or immobilizer for the first 6 weeks postoperatively. Of the protocols specifying mediumsized RTC tears (Fig 5B), $7(41.2 \%)$ recommended 6 weeks of postoperative sling or immobilizer use, with the second most prevalent recommendation, comprising 5 protocols $(29.4 \%)$, endorsing 4 weeks of use. Next, small-sized RTC tear protocols were compared (Fig 5C). Whereas the recommendations ranged from 2 to 6 weeks, the highest incidence was 6 protocols $(60 \%)$ recommending 6 weeks of use. Finally, protocols without specific RTC tear sizes were

\section{Transition to AROM Recommendations}

\section{A}
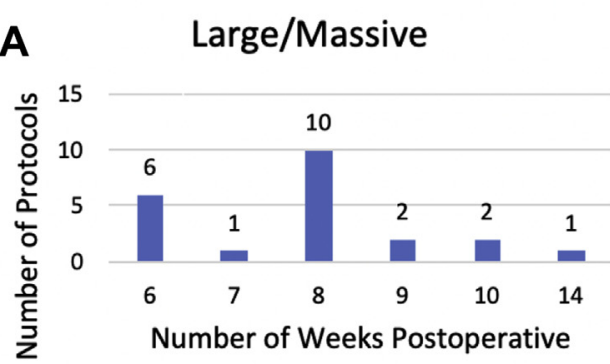

C

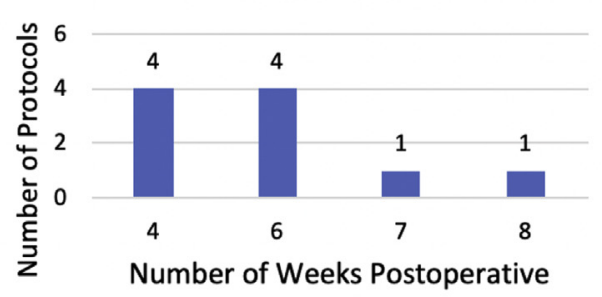

B

Medium

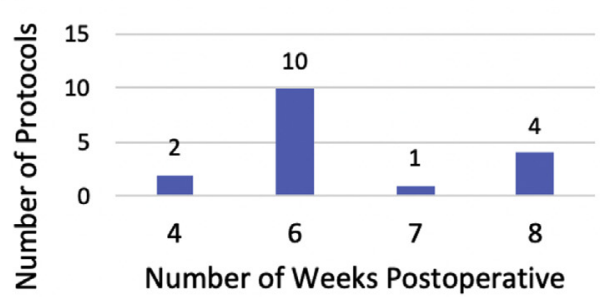

D

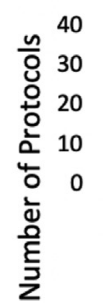

\section{Size Unspecified}

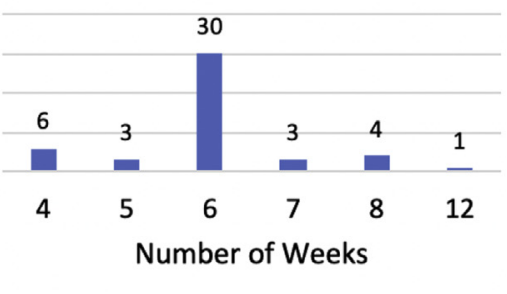

Fig 3. Recommendations for transition from passive range of motion to active range of motion (AROM) in protocols designated for large or massive (A), medium (B), and small (C) rotator cuff tears and protocols with rotator cuff size unspecified (D). 


\section{Recommendations for Return to Full ROM}

A

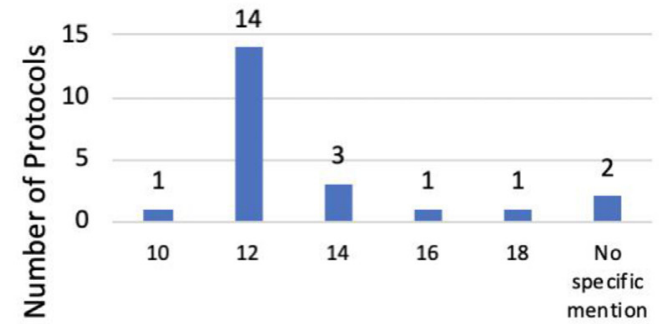

Number of Weeks Postoperative
B Medium

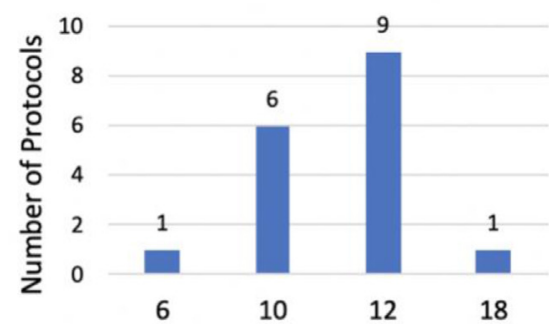

Number of Weeks Postoperative
C

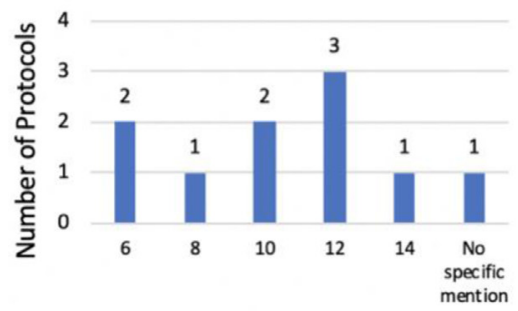

Number of Weeks Postoperative
D

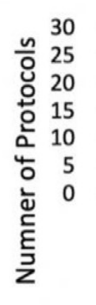

Size Unspecified

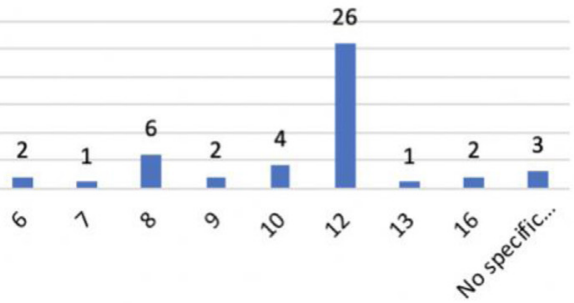

Number of Weeks
Fig 4. Recommendations for return to full range of motion ( $\mathrm{ROM})$ in protocols designated for large or massive (A), medium (B), and small (C) rotator cuff tears and protocols with rotator cuff size unspecified (D). compared (Fig 5D). The recommendations ranged from 3 to 8 weeks, but most protocols ( 31 protocols, $66.0 \%$ ) recommended sling or immobilizer use for the first 6 postoperative weeks. Overall, the predominant recommendation for time spent in a sling or immobilizer was 6 weeks, regardless of the size of the RTC tear.

\section{Recommendations for Sling/Immobilizer Use}

Fig 5. Recommendations for total time spent in a sling or immobilizer after repair of large or massive $(\mathrm{A})$, medium (B), and small (C) rotator cuff tears and in protocols with rotator cuff size unspecified (D).
A Large/Massive

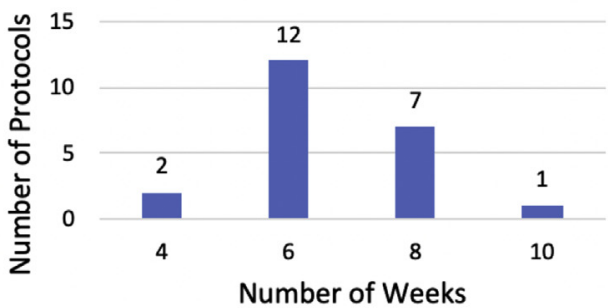

C

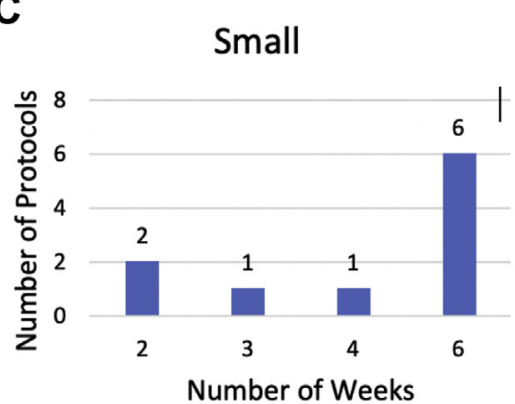

B Medium

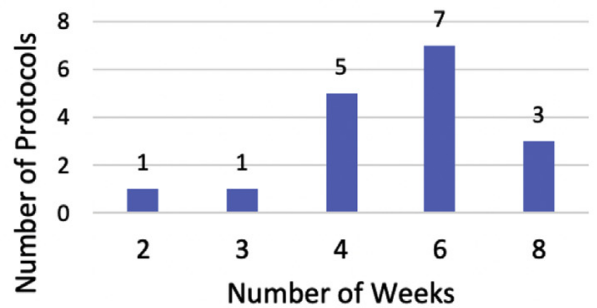

D Time Spent in a Sling/Immobilizer

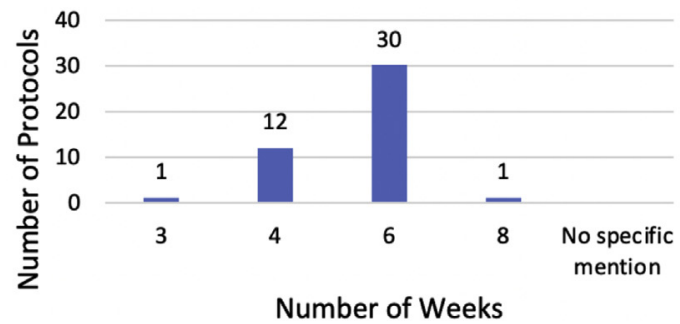




\section{Recommendations for Initiation of Postoperative Strengthening}

Fig 6. Recommendations for when to initiate postoperative shoulder or rotator cuff (RTC) strengthening in protocols for large or massive (A), medium (B), and small (C) RTC tears and protocols with RTC size unspecified (D).
A

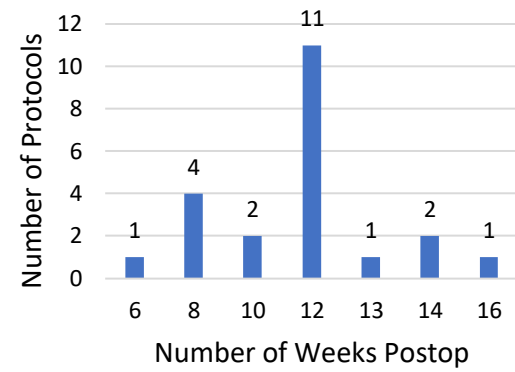

C

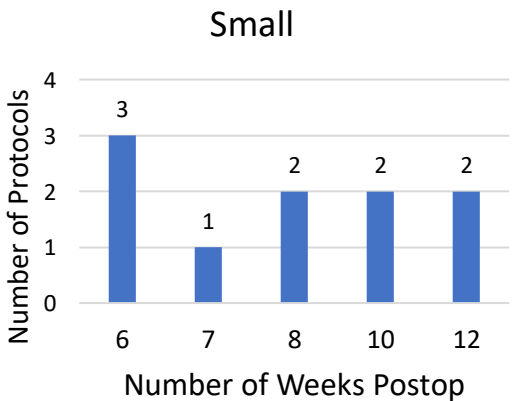

B Medium

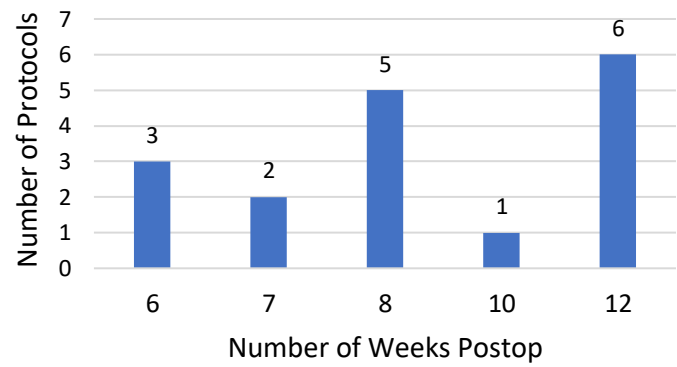

D

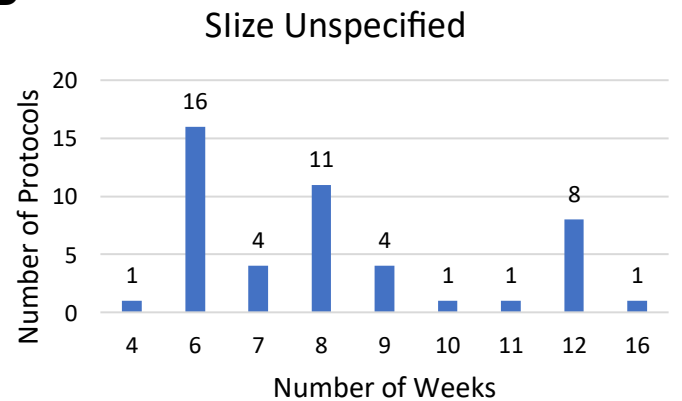

\section{Initiation of Formal Physical Therapy}

The recommended postoperative starting date for physical therapy was compared among the various protocols. Of the large to massive RTC tear protocols, 16 $(72.7 \%)$ did not indicate a specific postoperative starting date for physical therapy whereas $6(27.3 \%)$ did. Of these 6 protocols, $3(13.6 \%)$ recommended initiating physical therapy 4 weeks after surgery. The other 3 protocols recommended initiating physical therapy at anywhere from 1 to 6 weeks. Regarding medium-sized RTC tears, 14 of 17 protocols $(82.4 \%)$ did not make a specific recommendation for when to initiate postoperative physical therapy. Of the 3 protocols that did recommend a specific starting date, $2(11.8 \%)$ recommended initiation at 2 weeks and 1 (5.9\%) recommended initiation at 2 to 3 days postoperatively.

Regarding protocols designated for small RTC tears, 8 of 10 protocols $(80 \%)$ did not make specific recommendations for when to initiate postoperative physical therapy. Of the 2 protocols that did, $1(10 \%)$ recommended initiation at 2 to 3 days and $1(10 \%)$ recommended initiation at 2 weeks. Finally, physical therapy recommendations were compared in the 47 protocols without specific RTC sizes mentioned. Only 10 of these protocols $(21.3 \%)$ included recommendations for when to initiate physical therapy after surgery. Among these 10 protocols, the recommendations ranged from 1 day to 6 weeks, with the highest incidences being 4 protocols $(8.5 \%)$ recommending initiation at 1 week and 4 protocols $(8.5 \%)$ recommending initiation at 2 weeks.

\section{RTC Strengthening}

When to initiate postoperative shoulder and/or RTC strengthening was compared among the various rehabilitation protocols (Fig 6). All 22 large to massive RTC repair protocols recommended postoperative strengthening (Fig 6A). Of these protocols, 11 (50\%) recommended commencement of RTC strengthening at 12 weeks ( 3 months). The remaining 11 protocols $(50 \%)$ recommended commencement at anywhere from 6 weeks ( 1.5 months) to 16 weeks ( 4 months). All 17 medium RTC repair protocols recommended postoperative resistance strengthening of the shoulder or RTC (Fig 6B). However, the recommendations for return to strengthening in these protocols ranged from 6 to 12 weeks. The highest incidence was 6 protocols $(35.3 \%)$ recommending returning at 12 weeks ( 3 months), and the second highest was 5 protocols $(29.4 \%)$ recommending returning at 8 weeks (2 months).

All 10 small RTC repair protocols recommended postoperative resistance strengthening of the shoulder or RTC (Fig 6C). However, the recommendations 


\section{Recommendations for Return to Throwing}

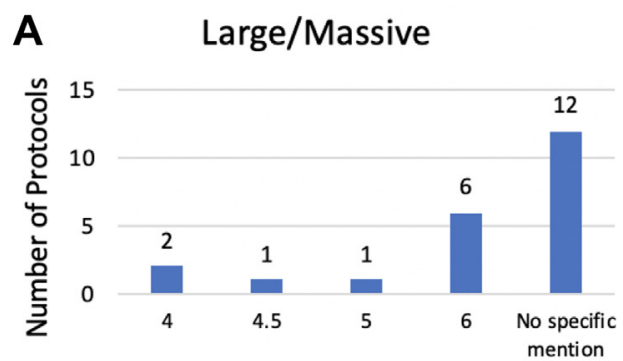

Number of Months

C

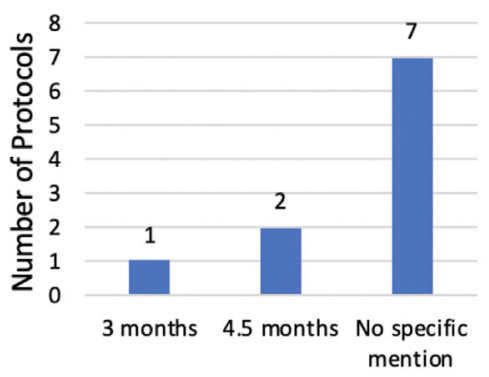

B

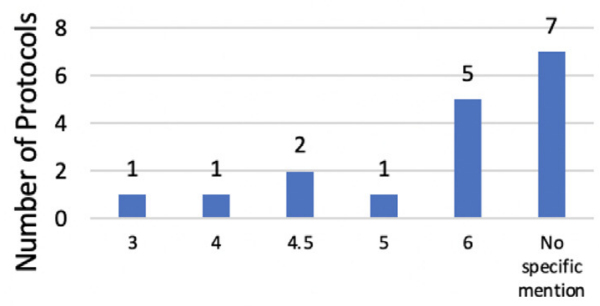

Number of Months

Fig 7. Recommendations for return to throwing in protocols for large or massive (A), medium (B), and small (C) rotator cuff tears and protocols with rotator cuff size unspecified (D).

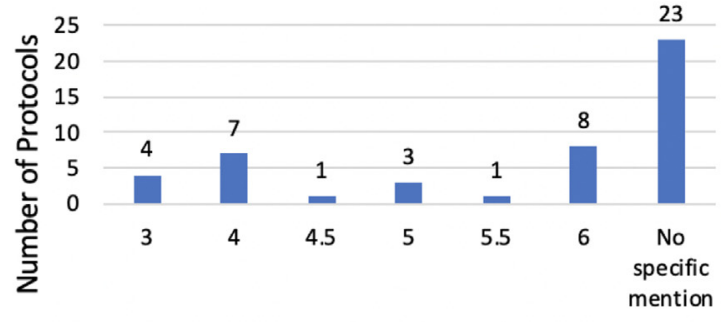

Number of Months ranged from initiation at 6 weeks to initiation at 12 weeks. The highest incidence was 3 protocols $(30 \%)$ recommending returning to strengthening at 6 weeks. Finally, the protocols without a specific RTC size were compared (Fig 6D). We found wide variation of recommendations in these protocols, ranging from 4 weeks ( 1 month) to 16 weeks (4 months) postoperatively. Of the protocols, $16(34.0 \%)$ recommended initiation at 6 weeks, $11 \quad(23.4 \%)$ recommended initiation at 8 weeks, and $8(17.0 \%)$ recommended initiation at 12 weeks.

\section{Return to Throwing}

Protocols were examined to determine whether they included criteria for return to a throwing program (Fig 7). Of the 22 protocols specific to large to massive RTC tears, $12(54.5 \%)$ did not mention returning to throwing whereas $10(45.5 \%)$ did (Fig 7A). Of these 10 protocols, $6(27.3 \%)$ recommended waiting until 6 months postoperatively before returning to throwing or initiating a throwing program. Of the 17 protocols specific to medium RTC tears (Fig 7B), 10 (58.8\%) had specific instructions for return to throwing. Of these 10 protocols, $5(29.4 \%)$ recommended returning to throwing at 6 months. The remaining 5 (29.4\%) recommended returning to throwing within the range of 3 to 5 months.
Of the 10 protocols specific to small RTC tears, only $3(30 \%)$ had specific instructions for return to throwing (Fig $7 \mathrm{C}) ; 2$ protocols $(20 \%)$ recommended initiation at 4.5 months, whereas $1(10 \%)$ recommended initiation at 3 months. Finally, of the 47 protocols without RTC sizes mentioned, 24 (51.1\%) made recommendations for return to throwing (Fig 7D). The recommendations ranged from 3 to 6 months, with the highest incidences being 8 protocols $(17.0 \%)$ recommending returning at 6 months and 7 protocols $(14.9 \%)$ recommending returning at 4 months. Overall, a time frame for returning to throwing was mentioned in 49 of the 96 protocols $(51.0 \%)$.

\section{Return to Sport}

Recommendations for RTS were compared among the various rehabilitation protocols (Fig 8). Of the large to massive RTC protocols (Fig 8A), 20 (90.9\%) made an RTS recommendation. Among these 20 protocols, there was a wide range of recommendations between 3 and 12 months (1 year), but the largest incidence of protocols (9 protocols, $40.9 \%$ ) recommended RTS at 6 months. In addition, 1 protocol $(4.5 \%)$ that recommended RTS at 6 months also specified no contact sports for 9 months after surgery. Regarding protocols specific to medium RTC tears (Fig 8B), 16 of 17 


\section{Recommendations for Return to Sport}

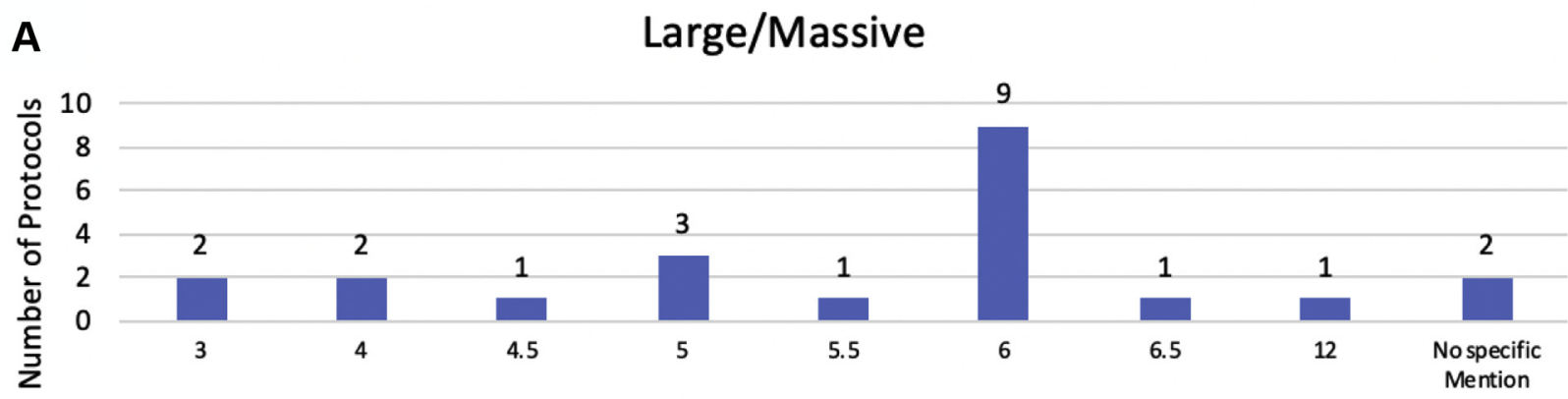

Number of Months

B

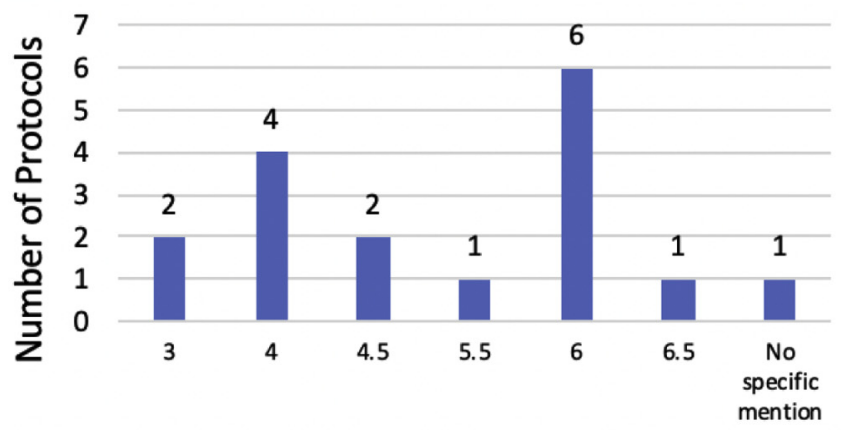

Number of Months
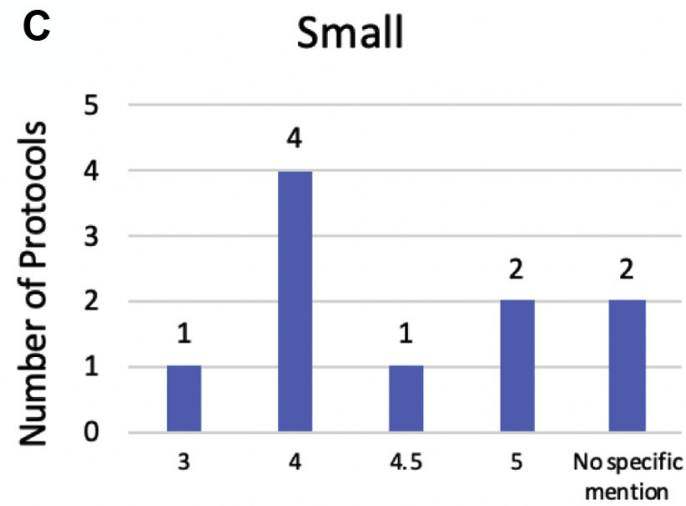

Number of Months

D Size Unspecified

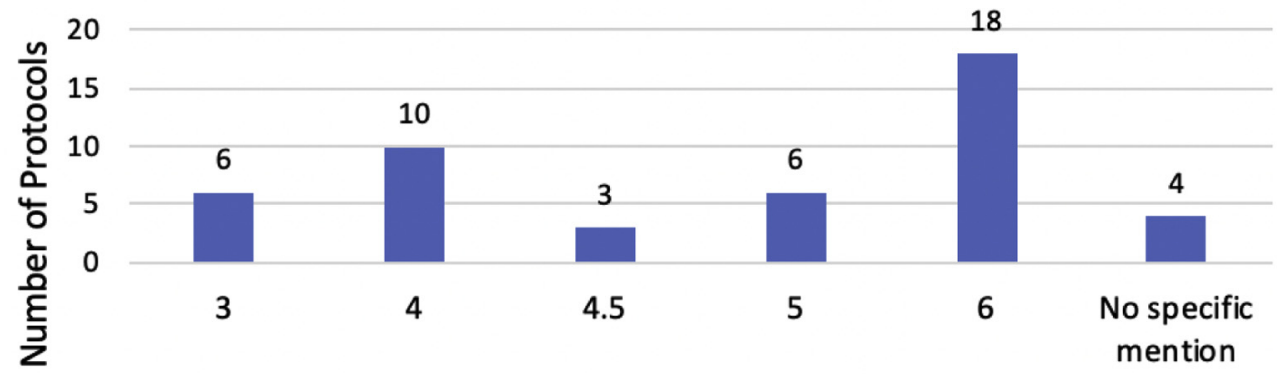

Number of Months

Fig 8. Recommendations for return to sport in protocols for large or massive (A), medium (B), and small (C) rotator cuff tears and protocols with rotator cuff size unspecified (D).

protocols $(94.1 \%)$ included instructions for RTS. The range of recommendations was between 3 and 6.5 months, but the highest incidence was 6 protocols $(35.3 \%)$ with a recommendation for RTS at 6 months. In addition, 1 protocol $(5.9 \%)$ that recommended RTS at 6 months also specified no contact sports for 9 months after surgery.
Regarding the small RTC protocols (Fig 8C), 8 of 10 $(80 \%)$ made specific recommendations for RTS. The recommendations ranged from 3 to 5 months, with the highest incidence being 4 protocols $(40 \%)$ recommending RTS at 4 months. Finally, 43 of the 47 protocols $(91.5 \%)$ without a specific RTC size mentioned made recommendations for RTS (Fig 8D). The 
recommendations ranged from 3 to 6 months, but the highest incidences were 18 protocols $(38.3 \%)$ recommending returning at 6 months and 10 protocols $(21.3 \%)$ recommending returning at 4 months. In addition, 5 protocols $(10.6 \%)$ specified no contact sports for 9 months after surgery. Of these 5 protocols, 3 $(6.4 \%)$ recommended RTS at 4 months, $1(2.1 \%)$ recommended RTS at 18 weeks, and $1(2.1 \%)$ recommended RTS at 6 months.

\section{Discussion}

This study showed that there is a lack of general consensus among the various rehabilitation protocols after an RTC repair. Although certain rehabilitation components were nearly always able to reach a majority agreement among protocols, such as time spent in a sling or immobilizer, others showed much more variation. For example, recommended time until return to resistance strengthening of the shoulder and duration of PROM were highly inconsistent among protocols. In addition, concern should be placed on the lack of instruction on when to initiate physical therapy. Only 24 of the 96 protocols $(25.0 \%)$ included specific instructions on when to initiate postoperative physical therapy. However, this may occur because physicians provide separate immediate postoperative instructions on when to initiate physical therapy, so this statistic is difficult to interpret. Future studies are needed to further assess when to start ROM with respect to RTC strengthening, as well as other specific rehabilitation stages.

Another topic of discussion is that 88 of the 96 protocols $(91.7 \%)$ did not place restrictions on early postoperative PROM, although doing so may actually be indicated. A consensus statement from the American Society of Shoulder and Elbow Therapists published by Thigpen et al. ${ }^{10}$ describes a rehabilitation framework that recommends an initial 2 -week period of strict immobilization, followed by introduction of PROM during postoperative weeks 2 to 6 . Furthermore, there is little evidence supporting the fact that early mobilization improves functional outcomes, ROM, pain, and retear rates when compared with conservative rehabilitation. ${ }^{11-14}$ In another study, Li et al. ${ }^{15}$ determined that at long-term follow-up, early passive motion may result in lower rates of tendon healing when it comes to large-sized tendon tears. Thus, with such a low consensus of protocols in this study $(91.7 \%)$ prohibiting early postoperative mobilization (no immediate restriction on PROM postoperatively), it is evident that further research on proper rehabilitation is needed. It is also worth noting that these instructions may vary based on the size of the tear because early mobilization may be more reasonably indicated for small or medium RTC tears. ${ }^{13,15}$ Moreover, as mentioned previously, it is difficult to distinguish whether physicians provide separate instructions (outside of the published rehabilitation protocols) regarding immediate postoperative ROM restrictions. This could be a limitation to this finding.

Regarding postoperative sling or immobilizer use, the small RTC tear protocol recommendations ranged from 2 to 6 weeks, with the highest incidence of recommendations being 6 protocols $(60 \%)$ recommending 6 weeks. Meanwhile, the recommendations for medium RTC tear protocols ranged from 2 to 8 weeks, with the highest incidence being 7 protocols $(41.2 \%)$ recommending 6 weeks of sling or immobilizer use. Overall, among the protocols designated for small ( 10 protocols) or medium ( 17 protocols) RTC tears, use of a postoperative sling or immobilizer was recommended for the first 4 weeks postoperatively or longer in 22 of 27 protocols $(81.5 \%)$. However, in a study looking at immobilization after RTC repair, Tirefort et al. ${ }^{16}$ found that sling use may not be indicated after repair of small or medium RTC tears, owing to their finding that undergoing no immobilization was associated with better early mobility and functional scores than undergoing sling immobilization. They also determined that undergoing no immobilization was significantly associated with reduced pain and improved function at 6 months compared with patients undergoing 4 weeks of sling use or immobilization. Considering that the most common complication after open or arthroscopic RTC repair is postoperative stiffness, ${ }^{8}$ these findings raise the question of whether sling or immobilization use should be recommended during postoperative rehabilitation after repair of small or medium RTC tears. However, without immobilization, there is greater concern about the risks of tendon retear and incomplete healing, so further research regarding the proper use and duration of sling wear is recommended.

Of the 96 rehabilitation protocols, $21(21.6 \%)$ recommended the use of cryotherapy postoperatively. In a study looking at the use of cryotherapy after shoulder surgical procedures (including RTC repair), Speer et al. ${ }^{17}$ determined that patients who received postoperative cryotherapy had a number of postoperative benefits. Some of these postoperative benefits included a reduced need for narcotics, better sleep, less swelling, and improved ability to participate during rehabilitation. However, there is evidence that the use of a standard ice pack with a wrap or ACE bandage ( $3 \mathrm{M}$ ) is just as effective as cryotherapy and is much more cost-effective, ${ }^{18,19}$ reducing the cost from $\$ 122.20$ (cryotherapy) to $\$ 6$ (standard ice pack). ${ }^{18}$ Although the use of standard ice packs may be more cost-effective, it comes with a risk of noncompliance. Further research regarding the use of cryotherapy versus standard ice packs in RTC rehabilitation protocols would be beneficial. 
This study illustrates that there is high variability in online postoperative rehabilitation protocols for RTC repair. We acknowledge that not every RTC tear is the same and there are many variables that determine how each patient should be treated. However, there is still a need for standardization of postoperative rehabilitation after RTC repair that takes into account the severity or size of the tear, patient's level of activity (occupation or sport type), and patient demographic characteristics. This standardization, in turn, would be more beneficial for providing patient education, decreasing the risk of confusion and, ultimately, helping improve patients' overall outcomes. Limited high-level evidence has illustrated how we should personalize or categorize a patient's postoperative rehabilitation protocol after RTC repair. For example, should we provide a specific therapy on the basis of the injury size, tissue quality, patient's age, patient's activity level, and/or sport type? Further studies are needed to help clarify this dilemma for our patients' care.

\section{Limitations}

Certain limitations to this study should be taken into consideration. Although a large number of protocols were included in this study, there are likely additional rehabilitation protocols that would have fit the inclusion criteria but were unable to be included because they have not been published online for public access. Furthermore, there are likely additional rehabilitation protocols published by private practice organizations that were not discovered during the electronic search. This is mainly because the Google search only included the names of academic institutions, not those of private practice organizations. Without a comprehensive list of national private practice organizations in the field of orthopaedics and sports medicine, an all-inclusive search of academic institutions and private practice organizations could not be completed without generating selection bias. Finally, one of the largest limitations of this study is that these online protocols may include many protocols based on lows levels of evidence. Although we would have preferred to investigate published, evidence-based rehabilitation protocols in this study, the sample size was too small to create a worthwhile study.

\section{Conclusions}

Although certain rehabilitation components were common, such as duration of PROM and sling or immobilizer use, a large degree of variation remains among published rehabilitation protocols after RTC repair, and this variability is still seen even when subdividing by the size or severity of the RTC tear.

\section{References}

1. Osborne JD, Gowda AL, Wiater B, et al. Rotator cuff rehabilitation: Current theories and practice. Phys Sportsmed 2016;44:85-92.

2. Rashid MS, Cooper C, Cook J, et al. Increasing age and tear size reduce rotator cuff repair healing rate at 1 year. Acta Orthop 2017;88:606-611.

3. Tashjian RZ. Epidemiology, natural history, and indications for treatment of rotator cuff tears. Clin Sports Med 2012;31:589-604.

4. Yamamoto A, Takagishi K, Osawa T, et al. Prevalence and risk factors of a rotator cuff tear in the general population. J Shoulder Elbow Surg 2010;19:116-120.

5. Seida JC, LeBlanc C, Schouten JR, et al. Systematic review: Nonoperative and operative treatments for rotator cuff tears. Ann Intern Med 2010;153:246-255.

6. Lee WH, Do HK, Lee JH, et al. Clinical outcomes of conservative treatment and arthroscopic repair of rotator cuff tears: A retrospective observational study. Ann Rehabil Med 2016;40:252-262.

7. Kuhn JE, Dunn WR, Sanders R, et al. Effectiveness of physical therapy in treating atraumatic full thickness rotator cuff tears: A multicenter prospective cohort study. J Shoulder Elbow Surg 2013;22:1371-1379.

8. Nikolaidou O, Migkou S, Karampalis C. Rehabilitation after rotator cuff repair. Open Orthop J 2017;11:154-162.

9. Mollison S, Shin JJ, Glogau A, et al. Postoperative rehabilitation after rotator cuff repair: A web-based survey of AANA and AOSSM members. Orthop J Sports Med 2017;5: 2325967116684775.

10. Thigpen CA, Shaffer MA, Gaunt BW, et al. The American Society of Shoulder and Elbow Therapists' consensus statement on rehabilitation following arthroscopic rotator cuff repair. J Shoulder Elbow Surg 2016;25:521-535.

11. De Roo PJ, Muermans S, Maroy M, et al. Passive mobilization after arthroscopic rotator cuff repair is not detrimental in the early postoperative period. Acta Orthop Belg 2015;81:485-492.

12. Keener JD, Galatz LM, Stobbs-Cucchi G, et al. Rehabilitation following arthroscopic rotator cuff repair: A prospective randomized trial of immobilization compared with early motion. J Bone Joint Surg Am 2014;96:11-19.

13. Mazuquin BF, Wright AC, Russell S, et al. Effectiveness of early compared with conservative rehabilitation for patients having rotator cuff repair surgery: An overview of systematic reviews. Br J Sports Med 2018;52: $111-121$.

14. Sheps DM, Silveira A, Beaupre L, et al. Early active motion versus sling immobilization after arthroscopic rotator cuff repair: A randomized controlled trial. Arthroscopy 2019;35:749-760.

15. Li S, Sun H, Luo X, et al. The clinical effect of rehabilitation following arthroscopic rotator cuff repair: A metaanalysis of early versus delayed passive motion. Medicine (Baltimore) 2018;97:e9625.

16. Tirefort J, Schwitzguebel AJ, Collin P, et al. Postoperative mobilization after superior rotator cuff repair: Sling versus no sling: A randomized prospective study. J Bone Joint Surg Am 2019;101:494-503. 
17. Speer KP, Warren RF, Horowitz L. The efficacy of cryotherapy in the postoperative shoulder. J Shoulder Elbow Surg 1996;5:62-68.

18. Dickinson RN, Kuhn JE, Bergner JL, et al. A systematic review of cost-effective treatment of postoperative rotator cuff repairs. J Shoulder Elbow Surg 2017;26:915-922.
19. Kraeutler MJ, Reynolds KA, Long C, et al. Compressive cryotherapy versus ice-A prospective, randomized study on postoperative pain in patients undergoing arthroscopic rotator cuff repair or subacromial decompression. J Shoulder Elbow Surg 2015;24:854-859. 